\title{
A Jacobson Radical Decomposition of the Fano-Snowflake Configuration
}

\author{
Metod SANIGA ${ }^{\dagger}$ and Petr PRACNA $\ddagger$ \\ † Astronomical Institute, Slovak Academy of Sciences, \\ SK-05960 Tatranská Lomnica, Slovak Republic \\ E-mail: msaniga@astro.sk \\ $\ddagger$ J. Heyrovský Institute of Physical Chemistry, v.v.i., Academy of Sciences of the Czech Republic, \\ Dolejškova 3, CZ-18223 Prague 8, Czech Republic \\ E-mail: pracna@jh-inst.cas.cz
}

Received July 14, 2008, in final form October 17, 2008; Published online October 24, 2008

Original article is available at http://www.emis.de/journals/SIGMA/2008/072/

\begin{abstract}
The Fano-Snowflake, a specific configuration associated with the smallest ring of ternions $R_{\diamond}$ (arXiv:0803.4436 and arXiv:0806.3153), admits an interesting partitioning with respect to the Jacobson radical of $R_{\diamond}$. The totality of 21 free cyclic submodules

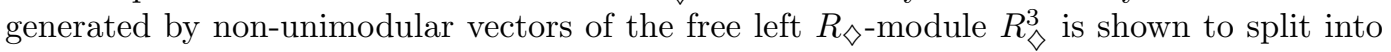
three disjoint sets of cardinalities 9,9 and 3 according as the number of Jacobson radical entries in the generating vector is 2,1 or 0 , respectively. The corresponding "ternioninduced" factorization of the lines of the Fano plane sitting in the middle of the FanoSnowflake is found to differ fundamentally from the natural one, i.e., from that with respect to the Jacobson radical of the Galois field of two elements.
\end{abstract}

Key words: non-unimodular geometry over rings; smallest ring of ternions; Fano plane

2000 Mathematics Subject Classification: 51C05; 51Exx

Projective lattice geometries over unital associative rings $R$ (see, e.g., [1] and references therein) represent a very important generalization of classical (field) projective spaces, being endowed with a number of remarkable features not exhibited by the latter. One of the most striking differences is, for certain $R$, the existence of free cyclic submodules generated by nonunimodular vectors of the free left $R$-module $R^{n+1}, n \geq 1$. In a couple of recent papers [2, 3], an in-depth analysis has been performed of such non-unimodular portions of the lattice geometries when $R$ is the ring of ternions, i.e., a ring isomorphic to that of upper triangular $2 \times 2$ matrices with entries from an arbitrary commutative field $F$. It has been found that for any $n \geq 2$ these non-unimodular free cyclic submodules of $R^{n}$ can be associated with the lines of $P G(n, F)$, the $n$-dimensional projective space over $F$ sitting in the middle of such a non-unimodular world. In the finite case, $F=G F(q)$, basic combinatorial properties of such configurations have been derived and illustrated in exhaustive detail for the simplest, $n=q=2$ case - dubbed the FanoSnowflake geometry. In the present paper we shall have another look at the Fano-Snowflake and show that this geometry admits an intriguing decomposition with respect to the Jacobson radical of the ring in question.

To this end, we first collect the necessary background information from $[2,3]$. We consider an associative ring with unity $1(\neq 0), R$, and denote the free left $R$-module on $n+1$ generators over $R$ by $R^{n+1}$. The set $R\left(r_{1}, r_{2}, \ldots, r_{n+1}\right)$, defined as follows

$$
R\left(r_{1}, r_{2}, \ldots, r_{n+1}\right):=\left\{\left(\alpha r_{1}, \alpha r_{2}, \ldots, \alpha r_{n+1}\right) \mid \alpha \in R\right\}
$$

is a left cyclic submodule of $R^{n+1}$. Any such submodule is called free if the mapping $\alpha \mapsto$ $\left(\alpha r_{1}, \alpha r_{2}, \ldots, \alpha r_{n+1}\right)$ is injective, i.e., if $\left(\alpha r_{1}, \alpha r_{2}, \ldots, \alpha r_{n+1}\right)$ are all distinct. Next, we shall call 
Table 1. Addition (left) and multiplication (right) in $R_{\diamond}$.

\begin{tabular}{||c|cccccccc||}
\hline \hline+ & 0 & 1 & 2 & 3 & 4 & 5 & 6 & 7 \\
\hline 0 & 0 & 1 & 2 & 3 & 4 & 5 & 6 & 7 \\
1 & 1 & 0 & 6 & 7 & 5 & 4 & 2 & 3 \\
2 & 2 & 6 & 0 & 4 & 3 & 7 & 1 & 5 \\
3 & 3 & 7 & 4 & 0 & 2 & 6 & 5 & 1 \\
4 & 4 & 5 & 3 & 2 & 0 & 1 & 7 & 6 \\
5 & 5 & 4 & 7 & 6 & 1 & 0 & 3 & 2 \\
6 & 6 & 2 & 1 & 5 & 7 & 3 & 0 & 4 \\
7 & 7 & 3 & 5 & 1 & 6 & 2 & 4 & 0 \\
\hline \hline
\end{tabular}

\begin{tabular}{||c|cccccccc||}
\hline \hline$\times$ & 0 & 1 & 2 & 3 & 4 & 5 & 6 & 7 \\
\hline 0 & 0 & 0 & 0 & 0 & 0 & 0 & 0 & 0 \\
1 & 0 & 1 & 2 & 3 & 4 & 5 & 6 & 7 \\
2 & 0 & 2 & 1 & 3 & 7 & 5 & 6 & 4 \\
3 & 0 & 3 & 5 & 3 & 6 & 5 & 6 & 0 \\
4 & 0 & 4 & 4 & 0 & 4 & 0 & 0 & 4 \\
5 & 0 & 5 & 3 & 3 & 0 & 5 & 6 & 6 \\
6 & 0 & 6 & 6 & 0 & 6 & 0 & 0 & 6 \\
7 & 0 & 7 & 7 & 0 & 7 & 0 & 0 & 7 \\
\hline \hline
\end{tabular}

a vector $\left(r_{1}, r_{2}, \ldots, r_{n+1}\right) \in R^{n+1}$ unimodular if there exist elements $x_{1}, x_{2}, \ldots, x_{n+1}$ in $R$ such that

$$
r_{1} x_{1}+r_{2} x_{2}+\cdots+r_{n+1} x_{n+1}=1 .
$$

It is a very well-known fact (see, e.g., $[4,5,6,7])$ that if $\left(r_{1}, r_{2}, \ldots, r_{n+1}\right)$ is unimodular, then $R\left(r_{1}, r_{2}, \ldots, r_{n+1}\right)$ is free; any such free cyclic submodule represents a point of the $n$-dimensional projective space defined over $R$ [5]. The converse statement, however, is not generally true. That is, there exist rings which also give rise to free cyclic submodules featuring exclusively non-unimodular vectors. The first case where this occurs is the smallest (non-commutative) ring of ternions, $R_{\diamond}$ :

$$
R_{\diamond} \equiv\left\{\left(\begin{array}{cc}
a & b \\
0 & c
\end{array}\right) \mid a, b, c \in G F(2)\right\}
$$

where the addition and multiplication is that of matrices over $G F(2)$. From this definition it is readily seen that the ring contains two maximal (two-sided) ideals,

$$
I_{1}=\left\{\left(\begin{array}{cc}
0 & b \\
0 & c
\end{array}\right) \mid b, c \in G F(2)\right\} \quad \text { and } \quad I_{2}=\left\{\left(\begin{array}{cc}
a & b \\
0 & 0
\end{array}\right) \mid a, b \in G F(2)\right\}
$$

which give rise to a non-trivial (two-sided) Jacobson radical $J$,

$$
J=I_{1} \cap I_{2}=\left\{\left(\begin{array}{ll}
0 & b \\
0 & 0
\end{array}\right) \mid b \in G F(2)\right\} .
$$

Since for our further purposes it will be more convenient to work with numbers than matrices, we shall relabel the elements of $R_{\diamond}$ as follows

$$
\begin{aligned}
& 0 \equiv\left(\begin{array}{cc}
0 & 0 \\
0 & 0
\end{array}\right), \quad 1 \equiv\left(\begin{array}{cc}
1 & 0 \\
0 & 1
\end{array}\right), \quad 2 \equiv\left(\begin{array}{ll}
1 & 1 \\
0 & 1
\end{array}\right), \quad 3 \equiv\left(\begin{array}{ll}
1 & 1 \\
0 & 0
\end{array}\right), \\
& 4 \equiv\left(\begin{array}{cc}
0 & 0 \\
0 & 1
\end{array}\right), \quad 5 \equiv\left(\begin{array}{cc}
1 & 0 \\
0 & 0
\end{array}\right), \quad 6 \equiv\left(\begin{array}{cc}
0 & 1 \\
0 & 0
\end{array}\right), \quad 7 \equiv\left(\begin{array}{cc}
0 & 1 \\
0 & 1
\end{array}\right) \text {. }
\end{aligned}
$$

In this compact notation the addition and multiplication in the ring reads as shown in Table 1. The two maximal ideals now acquire the form

$$
I_{1}:=\{0,4,6,7\} \quad \text { and } \quad I_{2}:=\{0,3,5,6\},
$$

and the Jacobson radical reads,

$$
J=I_{1} \cap I_{2}=\{0,6\} .
$$


There exist altogether 21 free cyclic submodules of $R_{\diamond}^{3}$ which are generated by non-unimodular vectors. Taking their complete list from [2] one sees that they can be separated into the following three disjoint sets

$$
\begin{aligned}
& R_{\diamond}(6,6,7)=R_{\diamond}(6,6,4) \\
& =\{(0,0,0),(6,6,7),(6,6,4),(6,6,0),(0,0,4),(6,6,6),(0,0,6),(0,0,7)\}, \\
& R_{\diamond}(6,7,6)=R_{\diamond}(6,4,6) \\
& =\{(0,0,0),(6,7,6),(6,4,6),(6,0,6),(0,4,0),(6,6,6),(0,6,0),(0,7,0)\}, \\
& R_{\diamond}(7,6,6)=R_{\diamond}(4,6,6) \\
& =\{(0,0,0),(7,6,6),(4,6,6),(0,6,6),(4,0,0),(6,6,6),(6,0,0),(7,0,0)\}, \\
& R_{\diamond}(0,6,7)=R_{\diamond}(0,6,4) \\
& =\{(0,0,0),(0,6,7),(0,6,4),(0,6,0),(0,0,4),(0,6,6),(0,0,6),(0,0,7)\}, \\
& R_{\diamond}(0,7,6)=R_{\diamond}(0,4,6) \\
& =\{(0,0,0),(0,7,6),(0,4,6),(0,0,6),(0,4,0),(0,6,6),(0,6,0),(0,7,0)\}, \\
& R_{\diamond}(6,0,7)=R_{\diamond}(6,0,4) \\
& =\{(0,0,0),(6,0,7),(6,0,4),(6,0,0),(0,0,4),(6,0,6),(0,0,6),(0,0,7)\}, \\
& R_{\diamond}(7,0,6)=R_{\diamond}(4,0,6) \\
& =\{(0,0,0),(7,0,6),(4,0,6),(0,0,6),(4,0,0),(6,0,6),(6,0,0),(7,0,0)\}, \\
& R_{\diamond}(6,7,0)=R_{\diamond}(6,4,0) \\
& =\{(0,0,0),(6,7,0),(6,4,0),(6,0,0),(0,4,0),(6,6,0),(0,6,0),(0,7,0)\}, \\
& R_{\diamond}(7,6,0)=R_{\diamond}(4,6,0) \\
& =\{(0,0,0),(7,6,0),(4,6,0),(0,6,0),(4,0,0),(6,6,0),(6,0,0),(7,0,0)\}, \\
& R_{\diamond}(4,6,7)=R_{\diamond}(7,6,4) \\
& =\{(0,0,0),(4,6,7),(7,6,4),(6,6,0),(4,0,4),(0,6,6),(6,0,6),(7,0,7)\}, \\
& R_{\diamond}(4,7,6)=R_{\diamond}(7,4,6) \\
& =\{(0,0,0),(4,7,6),(7,4,6),(6,0,6),(4,4,0),(0,6,6),(6,6,0),(7,7,0)\}, \\
& R_{\diamond}(6,4,7)=R_{\diamond}(6,7,4) \\
& =\{(0,0,0),(6,4,7),(6,7,4),(6,6,0),(0,4,4),(6,0,6),(0,6,6),(0,7,7)\}, \\
& R_{\diamond}(4,4,6)=R_{\diamond}(7,7,6) \\
& =\{(0,0,0),(4,4,6),(7,7,6),(6,6,6),(4,4,0),(0,0,6),(6,6,0),(7,7,0)\}, \\
& R_{\diamond}(4,6,4)=R_{\diamond}(7,6,7) \\
& =\{(0,0,0),(4,6,4),(7,6,7),(6,6,6),(4,0,4),(0,6,0),(6,0,6),(7,0,7)\}, \\
& R_{\diamond}(6,4,4)=R_{\diamond}(6,7,7) \\
& =\{(0,0,0),(6,4,4),(6,7,7),(6,6,6),(0,4,4),(6,0,0),(0,6,6),(0,7,7)\}, \\
& R_{\diamond}(0,4,7)=R_{\diamond}(0,7,4) \\
& =\{(0,0,0),(0,4,7),(0,7,4),(0,6,0),(0,4,4),(0,0,6),(0,6,6),(0,7,7)\}, \\
& R_{\diamond}(4,0,7)=R_{\diamond}(7,0,4) \\
& =\{(0,0,0),(4,0,7),(7,0,4),(6,0,0),(4,0,4),(0,0,6),(6,0,6),(7,0,7)\}, \\
& R_{\diamond}(4,7,0)=R_{\diamond}(7,4,0) \\
& =\{(0,0,0),(4,7,0),(7,4,0),(6,0,0),(4,4,0),(0,6,0),(6,6,0),(7,7,0)\},
\end{aligned}
$$




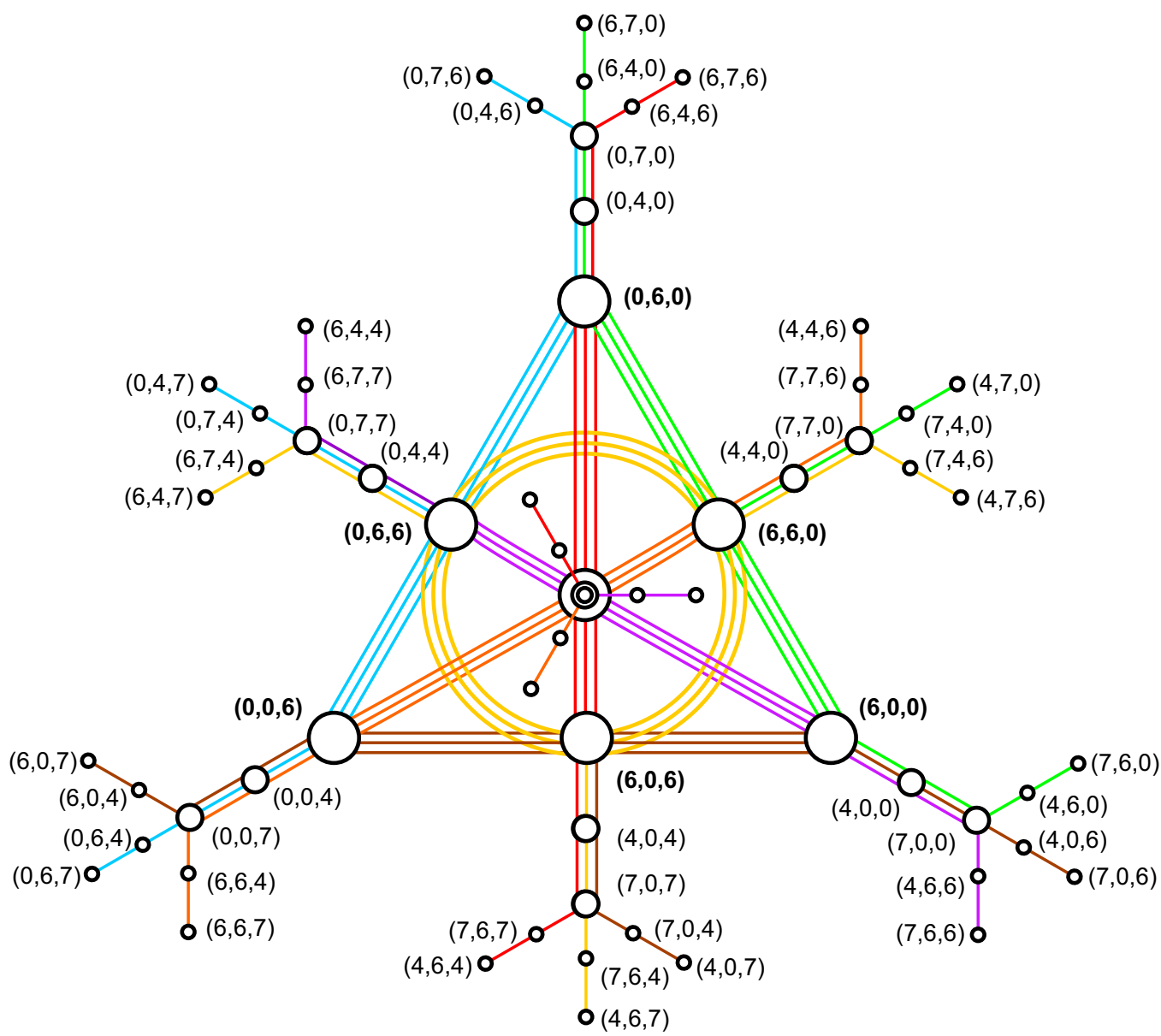

Figure 1. The Fano-Snowflake - a diagrammatic illustration of a very intricate relation between the 21 free left cyclic submodules generated by non-unimodular vectors of $R_{\diamond}^{3}$. Each circle represents a vector of $R_{\diamond}^{3}$ (in fact, of $I_{1}^{3}$ ), its size being roughly proportional to the number of submodules passing through the given vector. As the $(0,0,0)$ triple is not shown, each submodule is represented by seven circles (three big, two medium-sized and two small) lying on a common polygonal path. The small circles stand for the vectors generating the submodules. The big circles represent the vectors with all three entries from $J$; these vectors correspond to the points of the Fano plane. In the middle "branch" there are two medium-size circles in front of the big one; in order to avoid a too crowded appearance of the figure, these and the associated six small circles are not given the corresponding vector labels. The seven colors were chosen in such a way to also make the lines of the Fano plane, i.e., the intersections of the submodules with $J^{3}$, readily discernible. See [2] and/or [3] for more details.

$$
\begin{aligned}
R_{\diamond}(4,4,7) & =R_{\diamond}(7,7,4) \\
& =\{(0,0,0),(4,4,7),(7,7,4),(6,6,0),(4,4,4),(0,0,6),(6,6,6),(7,7,7)\}, \\
R_{\diamond}(4,7,4) & =R_{\diamond}(7,4,7) \\
& =\{(0,0,0),(4,7,4),(7,4,7),(6,0,6),(4,4,4),(0,6,0),(6,6,6),(7,7,7)\}, \\
R_{\diamond}(7,4,4) & =R_{\diamond}(4,7,7) \\
& =\{(0,0,0),(7,4,4),(4,7,7),(0,6,6),(4,4,4),(6,0,0),(6,6,6),(7,7,7)\},
\end{aligned}
$$

according as the number of Jacobson radical entries in the generating vector(s) is two, one or zero, respectively. Employing the picture of the Fano-Snowflake given in [2] (reproduced, for convenience, in Fig. 1), the structure of and relation between the three sets can be represented 

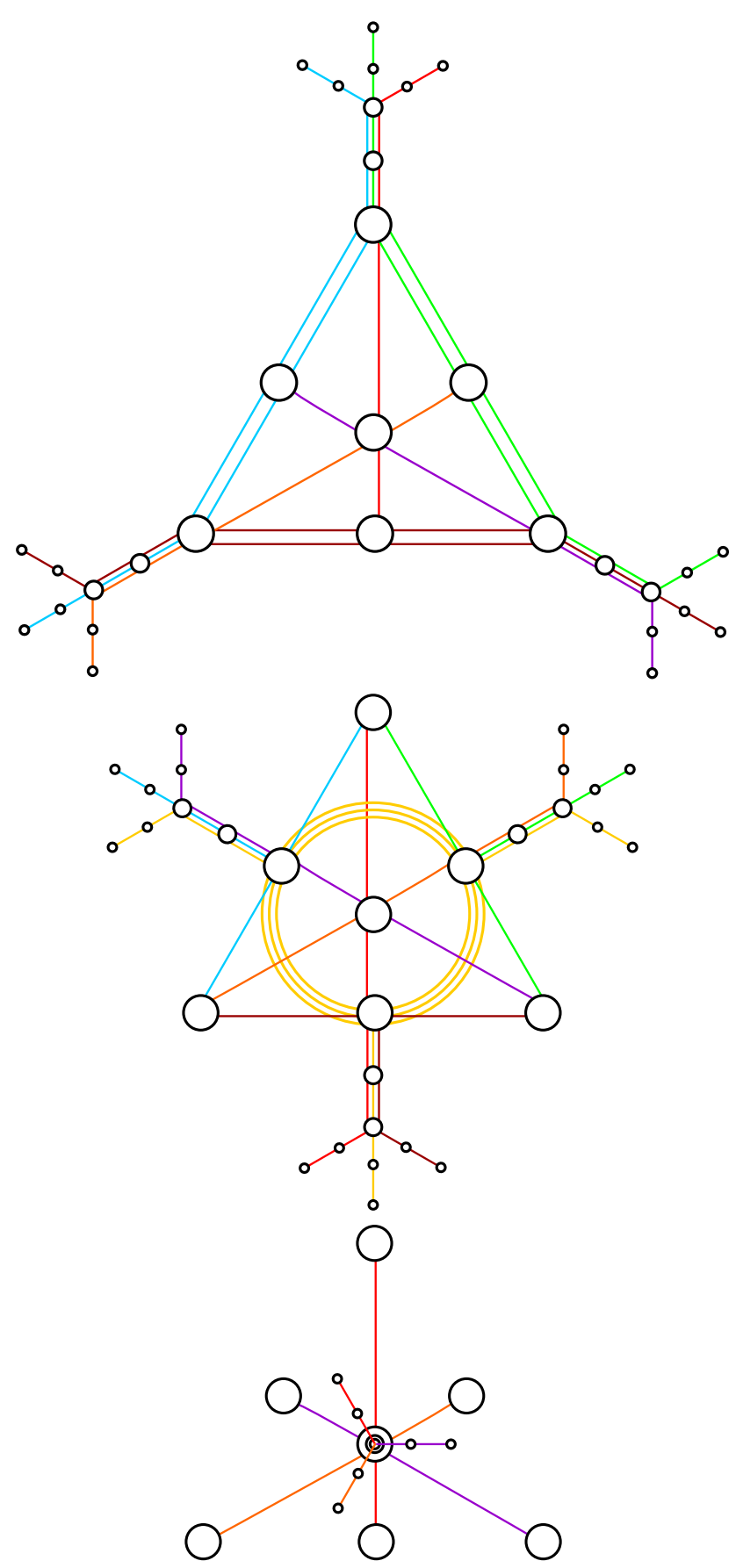

Figure 2. An illustration of the $9-9$ - 3 decomposition of the set of free cyclic submodules comprising the Fano-Snowflake with respect to the Jacobson radical of $R_{\diamond}$ according as the number of radical entries in the submodule's generating vector(s) is two (top), one (middle) or zero (bottom), respectively.

diagrammatically as shown in Fig. 2. As each submodule corresponds to a single line of the associated core Fano plane, the decomposition of the Fano-Snowflake induces an intriguing factorization of the lines of the plane itself. This is depicted in Fig. 3, bottom panel, and it is seen to fundamentally differ from the corresponding partitioning of the Fano plane with respect to the Jacobson radical of its ground field $G F(2)(\{0\})$, namely

$$
\begin{array}{ll}
G F(2)(1,0,0)=\{(0,0,0),(1,0,0)\}, & G F(2)(0,1,0)=\{(0,0,0),(0,1,0)\}, \\
G F(2)(0,0,1)=\{(0,0,0),(0,0,1)\}, & G F(2)(1,1,0)=\{(0,0,0),(1,1,0)\},
\end{array}
$$



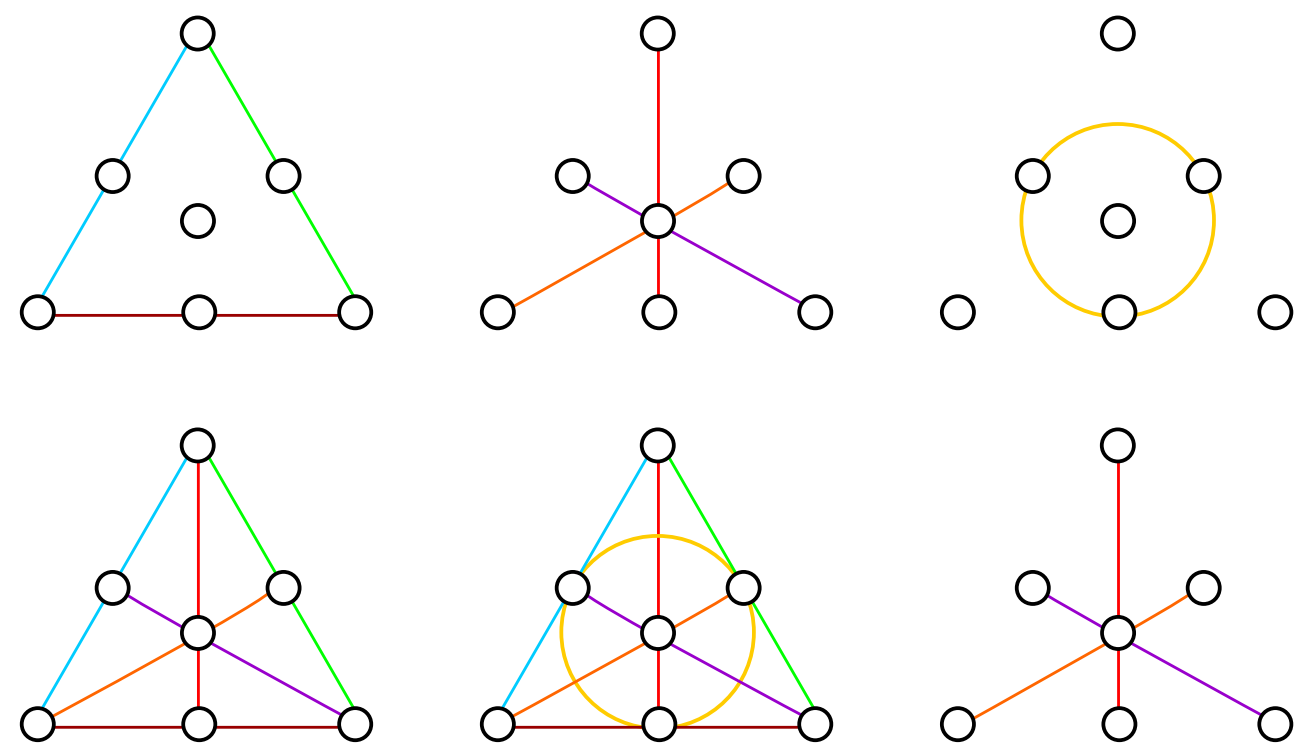

Figure 3. A comparison of the "ternion-induced" $6-7-3$ factorization of the lines of the Fano plane (bottom) with the ordinary $3-3-1$ one (top). Note a principal qualitative difference between the two factorizations, since the three sets (factors) are pairwise disjoint in the latter case but not in the former one.

$$
\begin{aligned}
& G F(2)(1,0,1)=\{(0,0,0),(1,0,1)\}, \quad G F(2)(0,1,1)=\{(0,0,0),(0,1,1)\}, \\
& G F(2)(1,1,1)=\{(0,0,0),(1,1,1)\},
\end{aligned}
$$

as displayed in Fig. 3, top panel.

The origin of the factorization of the Fano plane when related to its ground field $G F(2)$ is easy to understand: the number of the Jacobson radical entries (i.e., only zeros in this case) in the coordinates of a line (and, by duality, of a point as well) has a clear meaning with respect to the triangle of base points of the coordinate system. Something similar holds obviously for the factorization of the Fano-Snowflake with respect to its ternionic coordinates, but passing to the embedded Fano plane this link seems to be lost or substantially distorted. Fig. 2 illustrates this fact quite nicely: in the top figure the maximum number of polygonal paths pass through the three corners/vertices of the basic triangle, in the middle figure this property is enjoyed by the points on each side of the triangle which are not vertices, whereas in the bottom figure all the branches share the single point which is out of the reference triangle. It is the intersections of the branches/polygonal paths with the core Fano plane which behave "strangely" and give rise to the fundamental difference between the two factorizations of the Fano plane shown in Fig 3.

The observations above clearly demonstrate that there is more to the algebraic structure of the Fano plane than meets the eye. The plane when considered on its own is found to "reveal" quite different aspects compared with the case when embedded into a more general, non-unimodular projective lattice setting. This difference is likely to get more pronounced, and more intricate as well, as we pass to higher order rings giving rise to more complex forms of FanoSnowflakes. A key question is to find out whether the Snowflakes' decomposition patterns and their induced factorizations of the lines of the core Fano planes remain qualitatively the same as in the ternionic case; our preliminary analysis of such structures over a particular class of noncommutative rings of order sixteen and having twelve zero-divisors indicates that this might be so. Another line of exploration worth pursuing is to stay with ternions but focus on higher-order $(q>2)$ and/or higher-dimensional $(n>2)$ "Snowflake" geometries and their core projective planes and/or spaces. Finding, however, a general construction principle for these remarkable geometrical structures with respect to the properties of defining rings currently seems to be - 
already for the simplest $q=2$ case - a truly difficult, yet extremely challenging task due to the "ubiquity" of the Fano plane in various mathematical and physical contexts (see, e.g., [8]).

\section{Acknowledgements}

The work was partially supported by the VEGA grant agency projects Nos. 6070 and 7012, the CNRS-SAV Project No. 20246 and by the Action Austria-Slovakia project No. 58s2. We thank Hans Havlicek (Vienna University of Technology) for valuable comments and suggestions.

\section{References}

[1] Brehm U., Greferath M., Schmidt S.E., Projective geometry on modular lattices, in Handbook of Incidence Geometry, Editor F. Buekenhout, Elsevier, Amsterdam, 1995, 1115-1142.

[2] Saniga M., Havlicek H., Planat M., Pracna P., Twin "Fano-Snowflakes" over the smallest ring of ternions, SIGMA 4 (2008), 050, 7 pages, arXiv:0803.4436.

[3] Havlicek H., Saniga M., Vectors, cyclic submodules and projective spaces linked with ternions, arXiv:0806.3153.

[4] Veldkamp F.D., Projective planes over rings of stable rang 2, Geom. Dedicata 11 (1981), 285-308.

[5] Veldkamp F.D., Geometry over rings, in Handbook of Incidence Geometry, Editor F. Buekenhout, Elsevier, Amsterdam, 1995, 1033-1084.

[6] Herzer A., Chain geometries, in Handbook of Incidence Geometry, Editor F. Buekenhout, Elsevier, Amsterdam, 1995, 781-842.

[7] Blunck A., Herzer A., Kettengeometrien - Eine Einführung, Shaker Verlag, Aachen, 2005.

[8] Brown E., The many names of (7,3,1), Math. Mag. 75 (2002), 83-94. 